Proc. Indian Acad. Sci. (Chem. Sci.), Vol. 105, No. 6, December 1993, pp. 629-636.

(C) Printed in India.

\title{
Electron-nuclear cross-relaxation effect on the photochemical reaction of benzaldehyde as studied by CIDNP and DNP
}

\author{
YUZURU YAMAKAGE, QING-XIANG MENG, \\ SAMEH SAAD ALI, KIMINORI MAEDA and TOHRU AZUMI* \\ Department of Chemistry, Faculty of Science, Tohoku University, Sendai 980, Japan
}

\begin{abstract}
The magnetic field dependence of CIDNP was measured for the photolysis of benzaldehyde. At high and low fields, respectively, the CIDNP behavior is satisfactorily interpreted by the $S-T_{0}$ and $S-T_{-}$mixing of radical pair mechanism (RPM). At around $325 \mathrm{mT}$ the CIDNP of the aldehyde proton is emissive, which cannot be interpreted by RPM. In order to understand this anomalous behavior, we observe the dynamic nuclear polarization.

The DNP sign shows that the cross-relaxation mechanism of the intermediate free radical is $\Delta m=2$. With this result, the emissive CIDNP at $325 \mathrm{mT}$ is interpreted by the triplet mechanism (TM) with cross-relaxation-induced polarization transfer from electron spin to nuclear spin.

Further, the time-resolved DNP is observed with laser excitation and switching of microwaves. With pulse excitation, the features of CIDNP and DNP are different from that with continuous light excitation. This is interpreted as due to the associated increase of the initial concentration of the ketyl radical. From the time dependence of the DNP intensity, the lifetime of the ketyl radical is estimated to be of the order of hundreds of nanoseconds.
\end{abstract}

Keywords. CIDNP; CIDNP-detected ESR; DNP; cross-relaxation.

\section{Introduction}

The hydrogen abstraction reaction of benzaldehyde is one of the most interesting systems from the viewpoint of the CIDNP generation mechanism. A number of scientists (Closs and Paulson 1970; Cocivera and Trozzolo 1970; Atkins et al 1973; Frith and McLauchlan 1975) have studied the CIDNP with continuous light at high magnetic field, and the reaction scheme and the generation mechanism of the nuclear polarization have been thoroughly discussed. The time-resolved CIDNP has also been studied with laser excitation by Tsentalovich et al (1989) and the time-dependent feature of the free radical has been discussed. The CIDNP behavior reported so far is explained satisfactorily by RPM. Although the generation of CIDNP at higher fields in NMR is obvious, the CIDNP process at lower magnetic fields is not necessarily well understood.

As is discussed below, the CIDNP signal at a magnetic field of about $325 \mathrm{mT}$ is anomalously polarized. A plausible pathway for this anomaly appears to be the triplet mechanism (TM) (Adrian et al 1976; Meng et al 1993), in which polarization transfers from electron spin to nuclear spin via cross relaxation. In order to examine the

\footnotetext{
*For correspondence
} 
validity of the triplet mechanism in CIDNP, it is necessary to determine the mechanism of the cross-relaxation process $(\Delta m=0$ or $\Delta m=2)$. The DNP technique is suitable for this because the sign of DNP directly reflects the type of cross-relaxation process as reported in the system of benzoquinone (Meng et al 1993). The DNP spectrum is a component of CIDNP-detected ESR, which is obtained by monitoring the microwave-induced nuclear polarization of the reaction product with different external magnetic fields. The effect of the microwave irradiation on the intermediate radicals should include two kinds of components: dynamic nuclear polarization (DNP) and stimulated nuclear polarization (SNP) (Bagryanskaya et al 1986). DNP is a microwave effect on free radicals. The saturation of electron-spin transition of free radicals generates nuclear polarization via cross relaxation. Therefore, generation of DNP is a competition between cross-relaxation time and free-radical lifetime. The sign of DNP is independent of hyperfine lines, and directly reflects the cross-relaxation mechanism ( $\Delta m=0$ or $\Delta m=2)$. The SNP is an effect of the microwave transition on radical pairs. Here the sign of SNP depends on hyperfine lines and the shape of SNP should be either $E / A$ (emission at lower magnetic field and absorption at higher magnetic field) or $A / E$. We try to observe the CIDNP-detected ESR spectrum at around $325 \mathrm{mT}$ to determine the sign of DNP; i.e. cross-relaxation process.

The dynamics of free radicals is closely related to generation of CIDNP by spinpolarization transfer which competes with the quenching of free radicals. DNP phenomena directly reflect the dynamics of free radicals and cross relaxation. Therefore we observed the time-resolved DNP with the switching microwave technique in order to discuss the time scale of dynamics of free-radical and spinpolarization transfer processes.

\section{Experimental}

The experimental set-up for DNP measurement is shown in figure 1 . We used the flow system in order to carry out photoreaction in a variable JEOL RE-1X magnet, and the NMR spectrum was measured at high field in a JEOL JNM-FX100 NMR spectrometer. The sample was irradiated by a $500 \mathrm{~W}$ Hg-Xe arc lamp with a UV-D33S glass filter (which filters out $\lambda<330 \mathrm{~nm}$ ) in the X-band ESR cavity. The X-band microwave is amplified with a $20 \mathrm{~W}$ continuous wave TWTA. The sample was transferred to the NMR magnet within two seconds. The DNP intensity is recorded as

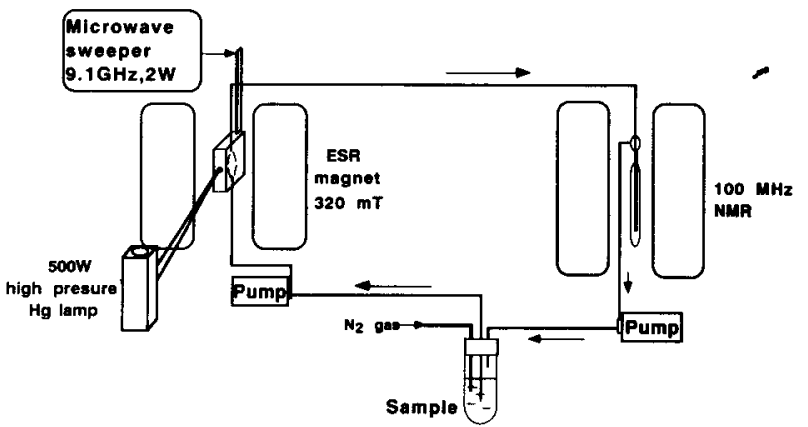

Figure 1. Block diagram of experimental set-up for DNP measurement. 
the difference of NMR integral with and without microwave irradiation. The microwave power used in the experiment was two watts.

The time-resolved DNP experiment was performed by the switching microwave technique using a PIN diode switch and laser excitation with a Lumonics EX-400 $(\mathrm{XeCl}, \lambda=308 \mathrm{~nm}$ ) excimer laser. Microwave-pulse duration was $1 \mathrm{~ms}$ which covered the lifetime of the free radical. The solution was deoxygenated by the nitrogenbubbling method. The solvent was a mixture of deuterated chloroform and carbon tetrachloride (volume ratio $1: 1$ ), and the concentration of benzaldehyde was $\approx 0.04 \mathrm{M}$.

\section{Results and discussion}

\subsection{CIDNP and DNP effect with continuous excitation}

In scheme 1, the hydrogen-abstraction reaction of benzaldehyde is outlined. The high field CIDNP spectrum was observed by a number of scientists (Closs and Paulson 1970; Cocivera and Trozzolo 1970; Atkins et al 1973; Frith and McLauchlan 1975) and the time-resolved CIDNP was observed by Tsentalovich et al (1989). The spectrum was completely explained by Kaptein's theory (Kaptein 1972) in terms of RPM with $S-T_{0}$ mixing. We observed the CIDNP spectrum (figure 2) at $325 \mathrm{mT}$ with variable magnet and flow system. The aldehyde proton of benzaldehyde exhibits emissive nuclear polarization. At such a high field as $325 \mathrm{mT}, S-T_{-}$and $S-T_{+}$mixings can be ignored. Therefore this emissive nuclear polarization of the aldehyde proton is anomalous from the viewpoint of RPM. This brings the effect of cross relaxation into our attention.

In order to determine the cross-relaxation mechanism, we need to observe the DNP effect. Since DNP appears as a component of the CIDNP-detected ESR spectrum, we have measured the CIDNP-detected ESR spectrum. The observed spectrum is shown in figure 3. The observation that the CIDNP-detected ESR spectrum is totally

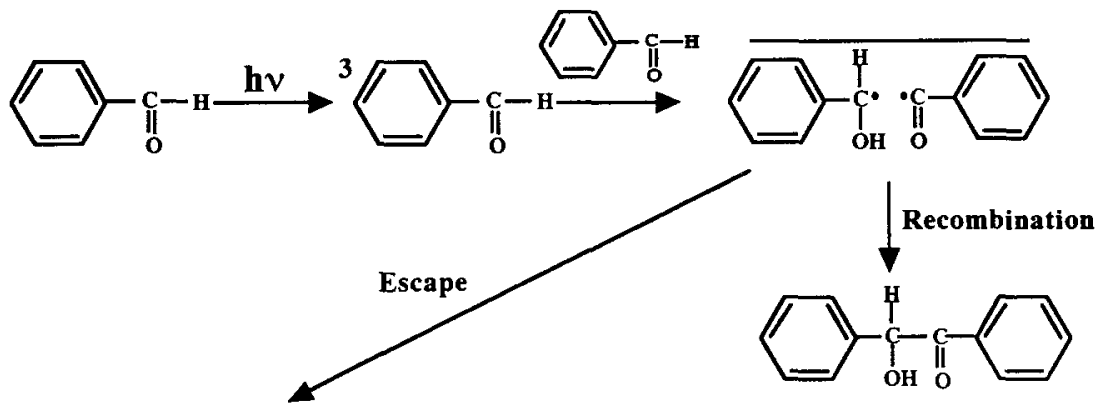

(a)

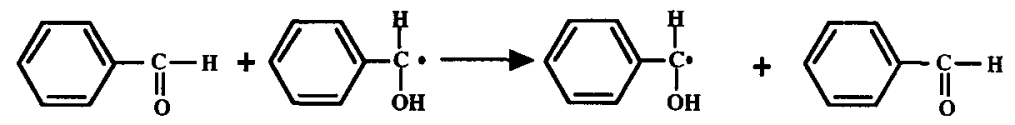

(b) 2

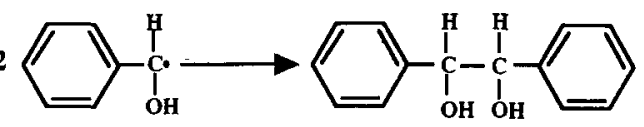

Scheme 1. Reaction scheme of photolysis of benzaldehyde in poor hydrogen donor solvent. 
(a)

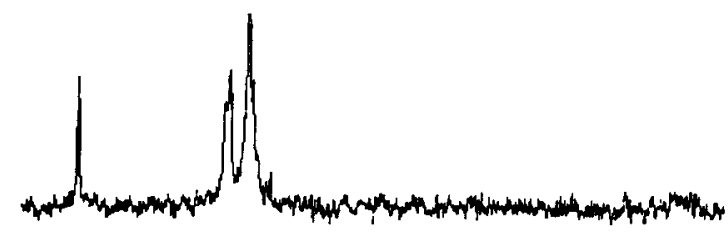

(b)

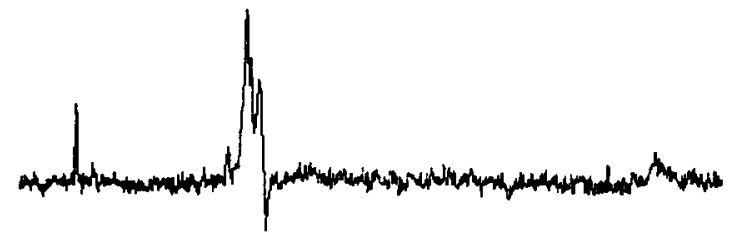

Figure 2. NMR spectra of benzaldehyde. (a) Before irradiation (b) CIDNP at $325 \mathrm{mT}$.

(a)

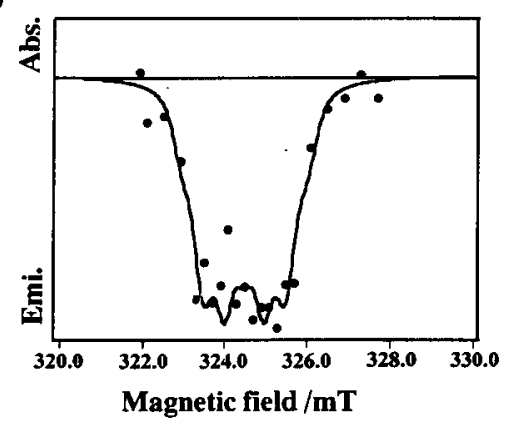

(b)

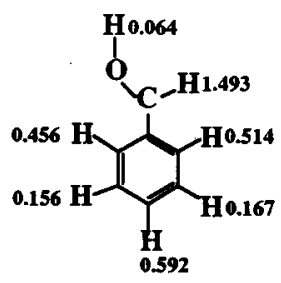

hyperfine coupling constant $/ \mathrm{mT}$

Figure 3. (a) CIDNP-detected ESR spectrum: Dots are experimental data, and solid line is simulated DNP spectrum using hyperfine coupling constants shown in (b).

emissive indicates that the SNP component is negligible, and we can therefore assign the observed spectrum as totally DNP. The solid line is the simulation using the hyperfine coupling constants reported by Wilson (1968). From the agreement between the observation and simulation, we conclude that the observed DNP spectrum is ascribed to the intermediate ketyl radical ( $\alpha$-hydroxybenzyl radical). This DNP spectrum is essentially the same as that reported by Grishin et al (1980).

The generation mechanism of DNP is shown in figure 4. The $\Delta m=2$ cross-relaxation process generates emissive DNP, and the $\Delta m=0$ generates absorptive DNP. Since the observed DNP is emissive, the cross-relaxation mechanism of the proton of ketyl radical which corresponds to the aldehyde proton of the reaction product (benzaldehyde) should be $\Delta m=2$. With the cross-relaxation process of $\Delta m=2$, we will discuss the generation mechanism of anomalous CIDNP signal at $325 \mathrm{mT}$. The ketyl radical is known to exhibit emissive CIDNP (Yamauchi and Hirota 1984), that is $\alpha$-electron spin states are populated predominantly. This electron-spin polarization can be transferred to nuclear polarization by cross relaxation. The same mechanism has been observed in the hydrogen-abstraction reaction of benzoquinone and some of its derivatives (Adrian et al 1976; Meng et al 1993), and has been referred to the triplet mechanism of CIDNP, which is shown in figure 5. The $\alpha$-electron spin states are initially more populated by the triplet mechanism. The $\Delta m=2$ cross relaxation 


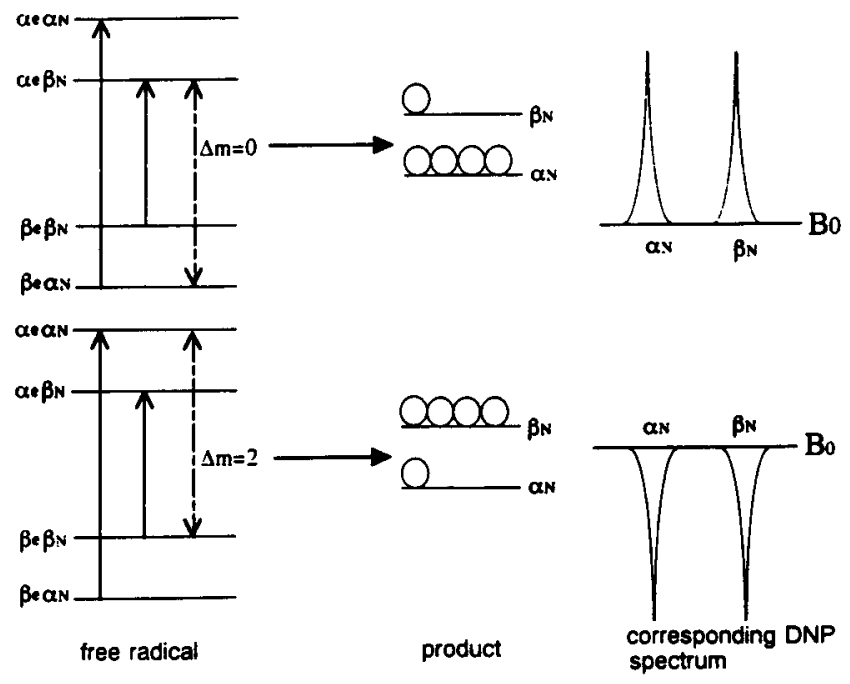

Figure 4. Schematic illustration of relationship between sign of DNP and crossrelaxation processes $(\Delta m=0$ and $\Delta m=2)$.

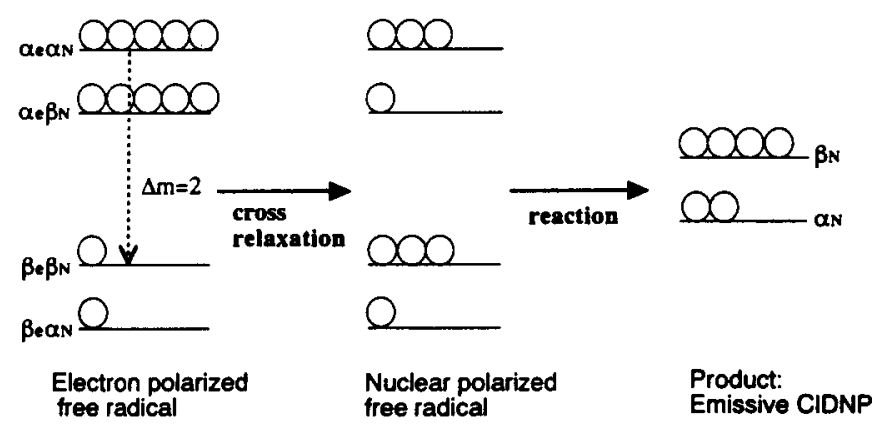

Figure 5. Generation mechanism of TM-induced CIDNP by $\Delta m=2$ cross-relaxation in the case of emissive CIDEP.

$\left(\alpha_{e} \alpha_{N}\right.$ to $\left.\beta_{e} \beta_{N}\right)$ transfers the emissive electron polarization to the nuclear polarization. The termination of the nuclear spin-polarized free radicals generates the emissive nuclear-polarized diamagnetic products, which is observed as anomalous CIDNP at $325 \mathrm{mT}$.

3.2 Results of DNP measurements with laser excitation and time-resolved DNP measurements

NMR spectra with laser excitation are shown in figure 6. The feature of CIDNP at $325 \mathrm{mT}$ is dramatically changed in comparison with the spectrum of continuous light excitation. As shown in figure $6 \mathrm{~b}$ the CIDNP of the aldehyde proton is weakly absorptive which is explained in terms of RPM. Additionally, the NMR signal of this proton is not affected by microwave irradiation (figure 6c). In contrast, we observe the microwave-induced emissive nuclear polarization at the aromatic protons. 
(a)

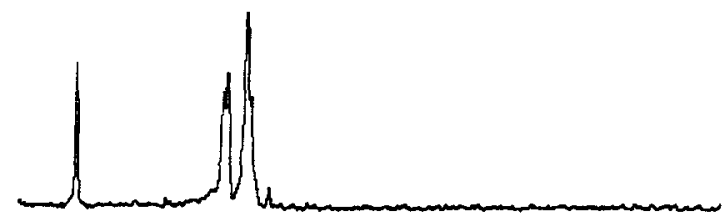

(b)

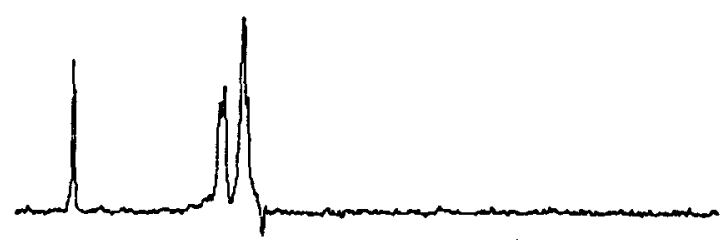

(c)

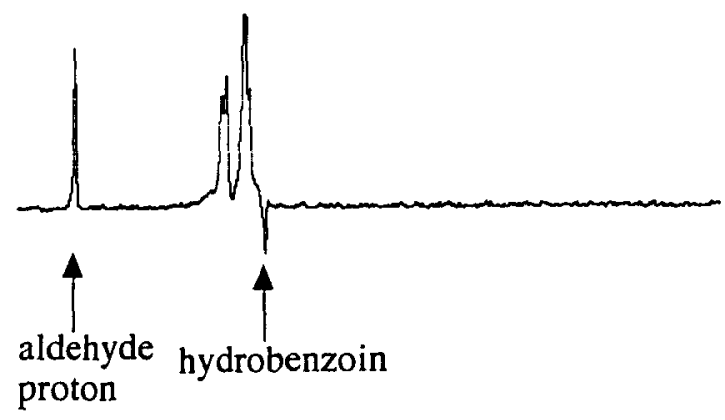

Figure 6. NMR spectra with laser excitation at $325 \mathrm{mT}$. (a) Before irradiation. (b) Irradiated by laser pulse (repetition rate is $15 \mathrm{~Hz}$ ). (c) With laser and pulsed microwave irradiation (microwave delay time is $-300 \mathrm{~ns}$ ).

This emissive nuclear polarization by microwave is DNP, not SNP, as is understood from the CIDNP-detected ESR spectrum, and the product which exhibits DNP is assigned as hydrobenzoin. The CIDNP signal of these protons was reported by Tsentalovich et al (1989) with time-resolved CIDNP technique at high magnetic field. In their experiment they also used an excimer laser whose pulse energy is about $40 \mathrm{~mJ}$. We should consider that much higher initial concentrations of ketyl radical caused by strong laser power affect the features of CIDNP and DNP. The decay of ketyl radicals is determined by the competition between the recombination of two ketyl radicals which generates hydrobenzoin (reaction (b) in scheme 1) and hydrogen transfer from the ketyl radical to benzaldehyde which generates nuclear-polarized benzaldehyde (reaction (a) in scheme 1). Using very simplified kinetic treatment we calculated the yield of two kinds of products, nuclear-polarized hydrobenzoin and benzaldehyde. We used reaction rate constants reported at time-resolved CIDNP study by Tsentalovich et al (1989). The initial radical-concentration dependence of the yields of two products is shown in figure 7 . The calculation shows that the hydrobenzoin should be dominant in case of very high initial concentration of ketyl radicals. Although it is difficult to estimate the initial concentration of the free radical exactly, the result of the calculation supports our experimental results qualitatively.

In order to estimate the lifetime of ketyl radicals with laser excitation, we measured the time-resolved DNP with switching microwave as shown in figure 8 . The time 


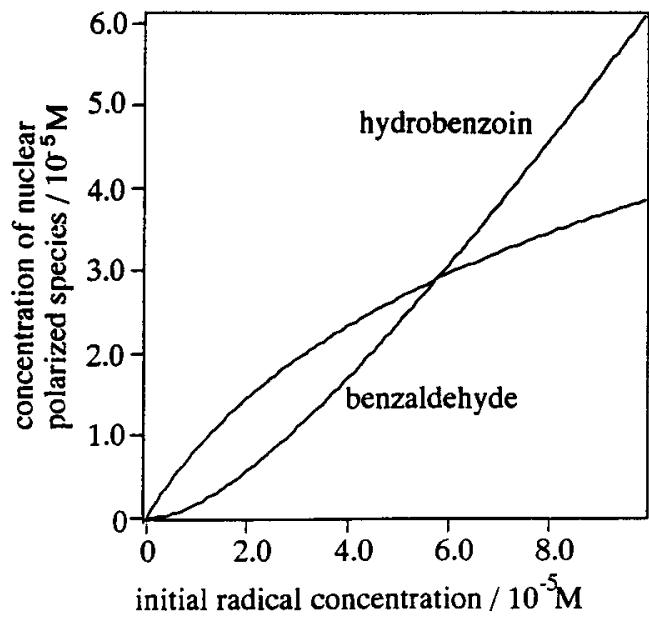

[ketyl radical] $\stackrel{K_{1}}{\longrightarrow}$ [benzaldehyde]

$2[$ ketyl radical] $\stackrel{\mathrm{K} 2}{\longrightarrow}$ [hydrobenzoin]

$\mathrm{K} 1=1.5 \times 10^{-6} \mathrm{M}^{-1} \mathrm{~s}^{-1} \times(0.04 \mathrm{M})$

$\mathrm{K}_{2}=1.3 \times 10^{-9} \mathrm{M}^{-1} \mathrm{~s}^{-1}$

Figure 7. Results of simple reaction kinetics equation. Nuclear-polarized product concentrations are modulated by initial radical concentration.

(a)

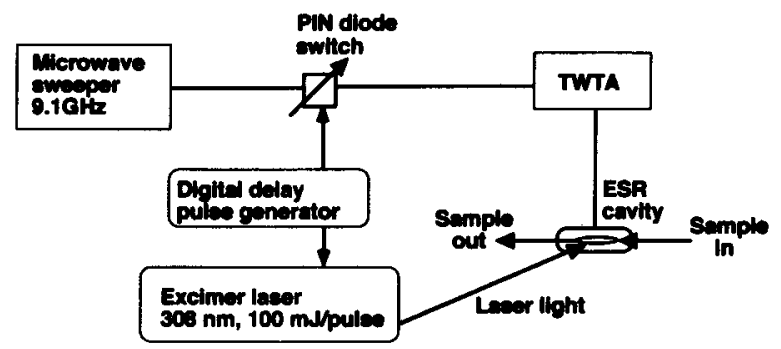

(b)

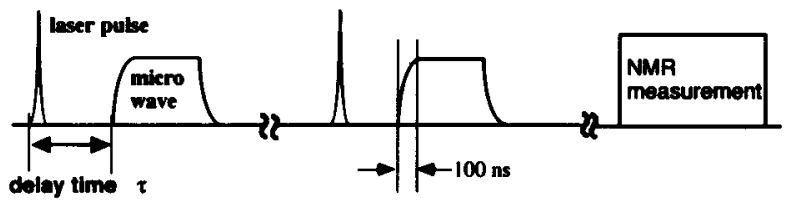

Figure 8. (a) Experimental set-up of time-resolved DNP. (b) Time sequence of excitation laser pulse and microwave pulse.

resolution of the time-resolved DNP measurement is determined by the response time of the microwave cavity, and is about $100 \mathrm{~ns}$ in our measurement. The delaytime dependence of DNP intensity (time-resolved DNP) with monitoring the aromatic protons of hydrobenzoin is shown in figure 9. The decay of the time-resolved DNP signal is about $250 \mathrm{~ns}$, and we conclude that the ketyl radical disappeared within 250 to $350 \mathrm{~ns}$ after laser excitation by taking the response time of the microwave cavity into account. Although the lifetime of ketyl radicals is less than $350 \mathrm{~ns}$, we could observe the DNP signal of the free radicals. This fact suggests a very fast polarization transfer of the aromatic protons of ketyl radicals from electron spin to nuclear spin. The time resolution of the time-resolved DNP is not enough to discuss the relation between reaction kinetics and dynamics of cross relaxation anymore. We are now developing a cavity with faster response time. 


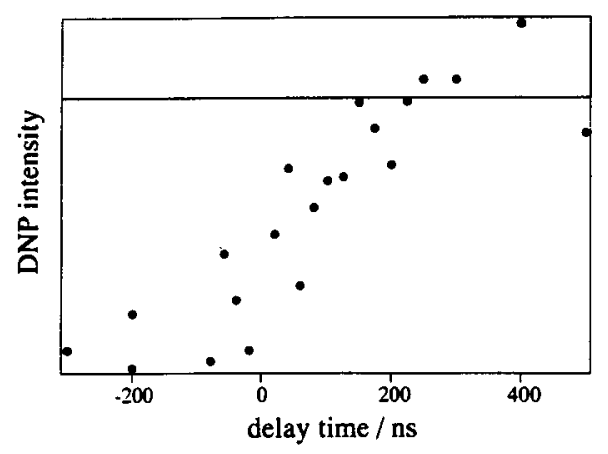

Figure 9. Delay-time dependence of DNP intensity monitoring aromatic protons of hydrobenzoin. Definition of delay time is shown in figure $8 \mathrm{~b}$.

\section{Conclusion}

The triplet mechanism-induced CIDNP should be taken into account for the analysis of the mechanism of CIDNP effect in addition to the radical pair mechanism. The sign of triplet mechanism-induced CIDNP is determined by the initial net electron polarization and the mechanism of cross relaxation $(\Delta m=0$ or $\Delta m=2)$. The former factor can be determined by time-resolved ESR, and the latter one can be directly determined by the DNP sign. The time-resolved DNP method can give information about the time dependence of intermediate radicals and the cross-relaxation processes.

\section{Acknowledgements}

The present research was supported by a Grant-in-Aid for Scientific Research No. 03554013, No. 0474027 and a Grant-in-Aid for Scientific Research on Priority Areas "Molecular Based Magnetism" No. 04242102 from Japanese Ministry of Education, Science and Culture.

\section{References}

Adrian F J, Vyas H M and Wan J K S 1976 J. Chem. Phys. 651454

Atkins P W, Frimston J M, Frith P G, Gurd R G and McLauchlan K A 1973 J. Chem. Soc., Faraday II 691543

Bagryanskaya E G, Tsentalovich Yu P, Avdievitch N I, Sagdeev R Z and Mglin Yu N 1986 Chem. Phys. Lett. 12

Closs G L and Paulson D R 1970 J. Am. Chem. Soc. 927229

Cocivera M and Trozzolo A M $1970 \mathrm{~J}$. Am. Chem. Soc. 921772

Frith P G and McLauchlan K A 1975 J. Chem. Soc., Faraday II 711984

Grishin Yu A, Gogolev A Z, Bagryanskaya E G, Dukushkin A V, Sagdeev R Z and Molin Yu N 1980 Dokl. Akad. Nauk. SSSR 2551160

Kaptein R 1972 J. Am. Chem. Soc. 946251

Meng Q X, Suzuki K, Maeda K, Terazima M and Azumi T 1993 J. Phys. Chem. 971265

Tsentalovich Yu, Obnochny A A and Sagdeev R Z 1989 Chem. Phys. Lett. 139301

Wilson R $1968 \mathrm{~J}$. Chem. Soc. B84 1581

Yamauchi S and Hirota N 1984 J. Phys. Chem. 884631 